\title{
Trimodal Therapy in Squamous Cell Carcinoma of the Esophagus
}

\author{
C. Matuschek ${ }^{1 *}$, E. Bölke ${ }^{1 *}$, T. Zahra ${ }^{1}$, W. T. Knoefel ${ }^{2}$, M. Peiper ${ }^{3}$, \\ W. Budach ${ }^{1}$, A. Erhardt ${ }^{4}$, A. Scherer ${ }^{5}$, S. E. Baldus ${ }^{6}$, P. A. Gerber ${ }^{1}$, B. A. Buhren ${ }^{1}$, M. Schauer ${ }^{2}$, \\ N.-Ph. Hoff ${ }^{1}$, N. Gattermann ${ }^{7}$, K. Orth ${ }^{8}$ \\ ${ }^{1}$ Klinik für Strahlentherapie und Radiologische Onkologie, Heinrich-Heine-Universität Düsseldorf, \\ ${ }^{2}$ Klinik für Allgemein, Viszeral- und Kinderchirurgie, Heinrich-Heine-Universität Düsseldorf, \\ ${ }^{3}$ Klinik für Allgemein-, Viszeral- und Unfallchirurgie, Kliniken Essen-Süd, Essen \\ ${ }^{4}$ Klinik für Gastroenterologie, Hepatologie und Infektiologie, Heinrich-Heine-Universität Düsseldorf, \\ ${ }^{5}$ Institut für Radiologie, Heinrich-Heine-Universität Düsseldorf, \\ ${ }^{6}$ Institut für Pathologie, Heinrich-Heine-Universität Düsseldorf, \\ ${ }^{7}$ Klinik für Hämatoonkologie, Onkologie und Klinische Immunologie, Heinrich-Heine-Universität Düsseldorf, \\ ${ }^{8}$ Klinik für Visceral- und Gefäßchirurgie, Klinikum Region Hannover, Germany
}

\begin{abstract}
Patients with ESCC (squamous cell carcinoma of the esophagus) are most commonly diagnosed with locally advanced tumor stages. Early metastatic disease and late diagnosis are common reasons responsible for this tumor's poor clinical outcome. The prognosis of esophageal cancer is very poor because patients usually do not have symptoms in early disease stages. Squamous cell carcinoma of the esophagus frequently complicates patients with multiple co-morbidities and these patients often require interdisciplinary diagnosis and treatment procedures. At present time, neoadjuvant radiation therapy and chemotherapy followed by surgery are regarded as the international standard of care. Meta-analyses have confirmed that this approach provides the patient with better local tumor control and an increased overall survival rate. It is recommended that patients with positive tumor response to neoadjuvant therapy and who are poor surgical candidates should consider definitive radiochemotherapy without surgery as a treatment option. In future, EGFR antibodies may also be administered to patients during therapy to improve the current treatment effectiveness. Positron-emission tomography proves to be an early response-imaging tool used to evaluate the effect of the neoadjuvant therapy and could be used as a predictive factor for the survival rate in ESCC. The percentage proportions of residual tumor cells in the histopathological analyses represent a gold standard for evaluating the response rate to radiochemotherapy. In the future, early response evaluation and molecular biological tests could be important diagnostic tools in influencing the treatment decisions of ESCC patients.
\end{abstract}

\section{INTRODUCTION}

In industrialized countries, squamous cell carcinoma of the esophagus has been shown to be frequently re-

\footnotetext{
* Both authors contributed equaly.

This work is dedicated to Kim and Thomas Speer.
}

lated to the abuse of alcohol and nicotine. This particular cancer is ranked as the sixth leading cause of all malignant tumors in the male population [1]. This may be due to the late diagnoses of the tumor and the tendency for early lymphatic spread. Unfortunately, the majority of these patients with esophageal carcinoma are diagnosed at an advanced tumor stage for ESCC (esophageal squamous cell carcinoma) as a consequence of less significant symptoms in early disease stages. ESCC is recognized as having a unique clinical behavior compared to adenocarcinoma of the esophagus [AEG type 2, Siewert classification] [2]. There are many key contrasting differences between these two types of tumors which are related to the tumor localization, pathogenesis, tumor biology and clinical outcome of the patient $[3,4]$. Single treatment modalities such as surgery or radiation therapy alone have been reported to have a poor prognosis for the patient and decreased overall survival. Furthermore, only one third of ESCC patients are surgically resectable after the primary staging results. Unfortunately, in the last thirty years, the clinical outcome and overall survival rate of these patients has demonstrated no improvement despite numerous trials performed to study the effectiveness of combination chemotherapy, radiation therapy and surgery. Studies which compared neoadjuvant multimodal treatment options with surgery alone had resulted in poor outcomes causing this approach to be considered controversial for many years [5]. In these studies, the approach was to compare treatment arm A with another treatment $\operatorname{arm}$ B. However, this approach had limited accuracy, and the results should be considered carefully [6]. After many published studies of patients with ESCC, it has been found that using a non-stratified mixture of patients consisting of various tumor stages, tumor locations and histological results will cause significant bias and may lead to incorrect results. This may be the reason for the inconsistent conclusions in older clinical studies. Today there has been a change of paradigms in the treatment of ESCC pa- 

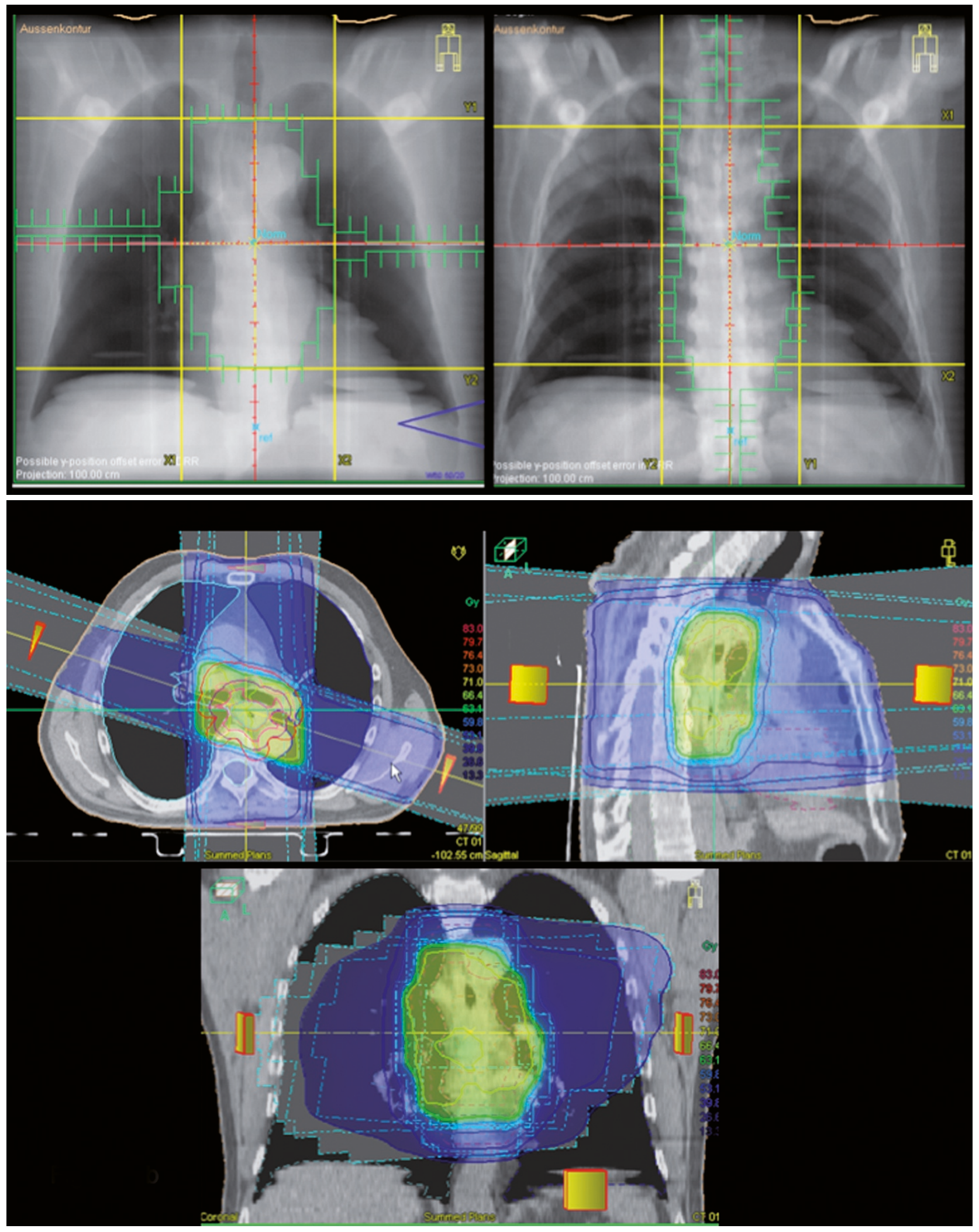

Fig. 1a-c. Treatment plan and dose distribution for 3-D conformal radiation therapy.

Fig. 2. PET-CT with a clear circular wall thickening in the middle section of the esophagus and increased glucose uptake (SUV max. 14,2). Prestenotic dilatation and bone metatasis (thoracic vertebrae 3 and 12). 

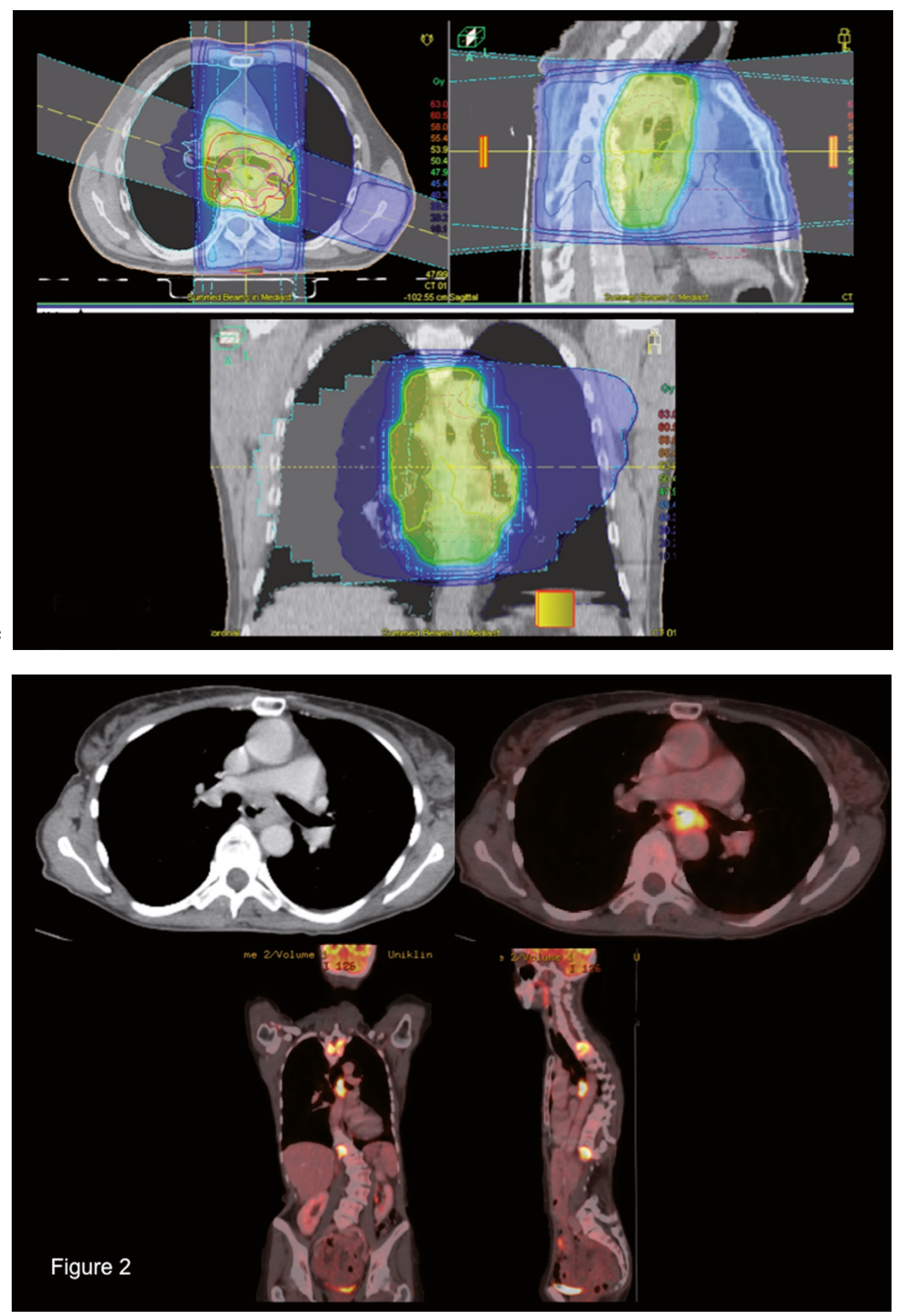
tients from the surgical resection to a multimodal treatment approach.

We want to address the different aspects of preoperative radio chemotherapy in ESCC patients and discuss this critically against the background of recent published studies.

\section{Primary Staging of the ESCC}

Endoscopy plays a critical role in the diagnosis and staging of ESCC with its ability to perform both biopsy and photo documentation. This diagnostic tool allows the physician to precisely visualize the location of the tumor. It is important for staging of the tumor that the examiner is able to distinguish between a cervical, supra bifurcal, or infra bifurcal location of the tumor, as well as to report involvement of the upper or inferior esophagus sphincter. In addition, it is essential for the surgeon to be notified about information regarding infiltration of the stomach, and regions of the pylorus and duodenum.

Due to the frequent submucosal tumor spread of ESCC, an endoluminal ultrasound (EUS) has been proven to play a very important role in diagnosing tumor size and stage. In addition to the tumor stage and lymph node status involvement playing a significant role in the diagnosis of the tumor, the location of the tumor with regard to the bronchial system ESCC is also a critical factor that should be considered. Infiltration of the tracheo-bronchial system is an important aspect to be considered in risk stratification and thorough examination using a bronchoscope with lavage or brush cytology is advised. Staging of the tumor with a computer tomogram of the thorax and abdomen is also advised. Some experts suggest the performance of an 18-FDG-positron emission computer tomography (PET-CT) to be beneficial [7]. A Doppler ultrasound is another useful imaging tool used for primary staging of the tumor to inspect for infiltration of the liver, and if suspicious findings are observed, a needle biopsy may then be performed.

Unfortunately, approximately $70 \%$ of all ESCC patients also tend to have comorbidity with COPD and nicotine. Of these patients with ESCC, 35\% are complicated by fatty liver disease or liver cirrhosis. Fur- thermore, malnutrition and a poor compliance are typical among these patients. This furthers the importance of discussing a safe and effective treatment for these patients. The final discussion of treatment options should be done in a multi-disciplinary tumor board where all clinical and laboratory results should be considered before a treatment method is decided. Only a multidisciplinary approach will provide a highly professional treatment decision tailored to the patient's individual clinical needs.

\section{Meta Analysis for Preoperative Radio CHEMOTHERAPY}

Published in a large number of randomized studies, including patients with squamous cell carcinoma, the overall survival rate of patients with combined preoperative radiochemotherapy followed by surgery has been shown to be significantly higher compared to patients with surgery alone (Table 1). However, the majority of the studies in the metaanalysis combined local resectable tumor stages (T3/T4) along with nonresectable tumors. These studies also included patients with tumors of various histology and may provide invalid results as there have been significant results proving that the tumor entity is responsible for the clinical outcome. ESCC patients receiving neoadjuvant therapy had a tumor mortality rate decreased by $27 \%$ (hazard ratio (HR) $0.84, \mathrm{p}=0.04$ ) over all studies and HR $0.75, \mathrm{p}=0.04$ in studies with radiochemotherapy simultanlously [13]. Patients receiving preoperative radiotherapy for $\mathrm{R} 0$ resections were found to have an increased success rate after therapy with an improvement of local tumor control.

The ESCC patients showed an increased rate of overall survival, although the postoperative mortality rate in some studies revealed to be increased by factor 2 ('Table 1). Patients receiving neoadjuvant therapy prior to surgical resection proved to be at an advantage as the prognosis with surgical resection alone is very poor [11]. As an implication of the results of these studies, the current gold standard for surgically resectable patients is to administer neoadjuvant radiochemotherapy followed by surgery.

Table 1. Metaanalysis of neoadjuvant radiochemotherapy vs. single surgical resection of esophagus cancer.

\begin{tabular}{lll}
\hline Study & Long time survival & Postoperative mortality rate \\
\hline Urschel 2003 [8] & HR 0.66 (0.47-0.92) $\mathrm{p}=0.02$ & HR 1.72 (0.96-3.07) $\mathrm{p}=0.07$ \\
Fiorica 2004 [9] & HR 0.53 (0.31-0.89) $\mathrm{p}=0.03$ & HR 2.10 (1.18-3.73) $\mathrm{p}=0.01$ \\
Malthaner 2004 [10] & HR 0.87 (0.80-0.96) $\mathrm{p}=0.004$ & \\
Stuschke 2004 [11] & HR 0.63 (0.47-0.85) $\mathrm{p}=0.002$ & HR 1.80 (1.10-2.90) $\mathrm{p}=0.02$ \\
Greer 2005 [12] & HR 0.86 (0.74-1.01) $\mathrm{p}=0.07$ & \\
Gebski 2007 [13] & HR 0.81 $(0.70-0.93) \mathrm{p}=0.002$ & \\
\hline
\end{tabular}

HR Hazard Ratio (95\% confidential interval) 


\section{StANDARDS IN THE PREOPERATIVE RADIO CHEMOTHERAPY}

The standard protocol for the application of preoperative radiochemotherapy is to administer a fractionated dose of 1.8 Gy daily to a total dose of 45 Gy . Following the last treatment of radiation, patients are then advised to proceed with surgical resection after a time interval of approximately 4-6 weeks. Induction chemotherapy is considered the standard of care in several cancer centers world-wide. The question that should be addressed is: Which chemotherapy should be given concurrently with radiation therapy? Many cancer centers frequently administer cisplatin and 5fluoruracil in lower concentration. There is also an option not to administer 5-FU to patients as this type of chemotherapy has been shown to increase the toxicity rate. A good alternative for chemotherapy treatment is to use a combination of cisplatin and taxan or irinotecan [14].

\section{REsponse Evaluation}

Several studies were performed to investigate the role of radiochemotherapy in patients with squamous cell carcinoma of the esophagus. The final results of these trials revealed a positive tumor response to neoadjuvant therapy signifying the importance of this treatment and could become a prognostic factor for patients. In a trial performed by the German Study Group of Esophageal Carcinoma it was reported that a patient responding to a high dose of radiochemotherapy without surgery had a statistically equivalent overall survival rate compared to patients receiving preoperative radiochemotherapy followed by surgical resection $[15,16]$. This result demonstrated no difference in overall survival with or without surgical resection and is especially important for patients who are at high risk for surgery and surgical resection is not recommended. Although the trial performed by the German Study Group recognized a benefit for patients receiving neoadjuvant therapy, the benefit was not related to patients that did not respond to neoadjuvant therapy. In future trials, a better selection of these non-responding patients may help to avoid ineffective and expensive treatment options. Induction chemotherapy in a patient with no response to neoadjuvant therapy may prove to be detrimental to a potential curative treatment by surgical resection. This study is especially important for patients who are good surgical candidates, a distal tumor location, and a surgically resectable tumor stage. The results of the German Study Group was verified by and compared with a French multi center study, in which $80 \%$ of patients with locally advanced squamous cell carcinoma of the esophagus were included [17].

After initial treatment with radiochemotherapy, the patients that responded to neoadjuvant treatment were then randomized into two different treatment arms. One treatment arm was administered surgery while the other treatment arm was administered radiation therapy and chemotherapy alone for a total of 18.5 months. The median survival rate for all responders in both treatment arms was shown to be independent of the treatment given and the results proved to have identical survival curves. The French multi center study reported that a patient with no response to neoadjuvant therapy had a decreased survival rate compared to the responders. The survival rate was statistically significant showing a decrease of 11.5 months compared to the responders $(p<0.002)$. This study documented that 112 patients out of a total of 192 non-responder patients $(58 \%)$ received surgical resection even though they had no regression of their tumor. These patients were found to have a median survival rate of 17.5 months which was similar to the results found when compared to the responders $(\mathrm{p}=0.59)$. The median survival for a non-responder without surgery was reported to be less than ten months [17]. The German and French study both reported an increased ability for local tumor control, which was defined by the patient's ability to eat meals until the end of life, following surgical resection of the tumor [15-17]. Some of the non-reponder were successfully treated by surgery. When a conservative approach of treatment with radiochemotherapy without surgery was taken, the local stenosis rate was $60 \%$ but with low morbidity. Without surgery there are $20 \%$ more locoregional recurrences which are responsible for dysphagia. At the same time there is a risk factor for radiotherapy to develop non malignant stenosis.

In the future, with the goal to improve local tumor control, treatment may be optimized with radiation therapy. This can be accomplished by increasing the prescribed tumor dose along with the advanced technology of intensive modulated radiation therapy. Planning of the dose distribution may also be improved with the application of PET-CT.

\section{Clinical Judgements of the Therapeutic RESPONSE}

The National Cancer Institute and the European Association for Research and Treatment of Cancer defined the clinical response of solid tumors using a one dimensional method. This method defined a regression of the greatest tumor diameter of $30 \%$ to be comparable to a spherical lesion with a regression of $50 \%$ [18].

It is important to recognize that when evaluating for tumor response to neoadjuvant treatment with the help of imaging and clinical procedures such as endoscopy, biopsy, endoluminal ultrasound, X-ray, and MNR procedures, the results are often unreliable and difficult to interpret. The ability to distinguish between a therapeutically induced scar tissue, fibrosis and histological residual tumor cells may be difficult [6]. Additional biopsies after multi-modal therapy approach are often not helpful due to the presence of response heterogeneity in the treated area. This may lead to false positive results and an incorrect report of positive tumor remission. It is possible for tumor cells to be located in close proximity to the biopsy site. This may be a result of different tumor reactions as well as centripetal reductions of the tumor as well as centrifugal tumor reaction with necrosis in the tumor center. To prevent a clinical judgement error, a distinguished point of view is advised. A radiology approach to 
down staging a reduction in a radio morphological tumor volume is known as a radiological judgement. The special effect of radiation therapy in a multi-modal approach is recognized by the direct effect on the tumor. It is important to recognize that radiotherapy may lead to swelling of the esophageal wall creating the appearance of an objective increase in tumor volume. This false interpretation visualized by computer tomogram imaging may influence the physician's clinical judgement. Therefore, it has been stated that an objective evaluation of a reduction in preoperative tumor volume is only reliable with the application of chemotherapy [19]. However, there are other research groups who do not support this conclusion [20, 21]. A perfusion computer tomogram may help to visualize the tumor more accurately and improve the clinical judgement made by the physician [22]. An additional disadvantage to using computer tomogram for clinical evaluation is the increased time interval which must be followed for the test to be performed. Clinical evaluation using computer tomogram is appropriate only after 4-6 weeks of treatment have been completed.

\section{Histopathological Approach}

Only after the tumor resection has been completed the histopathological regressions rate can be determined. This is the gold standard due to fact that only the pathologist can examine possible residual tumor infiltrations that may be present under re-epitheliazed tissue. Besides the evaluation of the ypTNM status after UICC [23], it is possible to define the pathological response rate according to the French pathologist Anne Marie Mandard [24]. The so-called regression grade is defined by a histopathological criteria, however, there are various regression gradings $[25,26]$. Becker et al. defined the criteria for regression to be divided as complete $(0 \%$ vital tumor cell), partial $(10-50 \%$ vital tumor cells) and little remission ( $>50 \%$ vital tumor cells) [27].

Meanwhile more than 50 non-randomized studies have been published with pathologic complete responses (pCR) ranging from 8 to $56 \%$

An investigation including 311 ESCC patients treated with multi-modal radiochemotherapy followed by esophageal resection demonstrated that the histopathological response rate can be determined by a simple and efficient method by using the application limit of $\leq 10 \%$ residual tumor cells [28]. It was reported that a histopathological responder $(<10 \%$ residual tumor cells) resulted in a significantly increased survival rate compared to a non responder $(>10 \%$ residual tumor cells). Furthermore, these investigations demonstrated that patients with a subtotal regression $(<10 \%$ residual tumor cells) had similar survival rates when compared to patients with complete regression ( $0 \%$ residual tumor cells).

\section{Metabolic Response}

At the present time, 18-FDG-PET (Fluor-18-Deoxyglucose positron emissions tomography) is discussed as diagnostic tool and may be used as a predictive imaging test showing similar correlation with the histopathological response of ESCC patients [6]. The
18-FDG-PET imaging study is measured by reporting the standard uptake values (SUV) which is the measurement of glucose uptake by the primary tumor. The test is generally performed prior to and after the beginning of a neoadjuvant therapy to safely show a comparison of the SUV values and verify if a tumor is responding to treatment $[29,30]$. The sensitivity of 18-FDG-PET imaging may be increased by application in combination with special markers like GLUT-1 [31]. Although using 18-FDG-PET testing has been shown to be useful for the majority of solid tumors, there are few solid tumors, for example those with extended lymphangiosis carcinomatosa, that do not metabolize 18-FDG. As a result, such tumors will not show increased uptake values and 18-FDG-PET should not be used to monitor tumor activity in these patients. In this context further studies are needed to verify the validity of this method.

The aim for the future is a diagnostic tool to determine who should be operated and who should be continued with radiation therapy in the neoadjuvant setting.

\section{Surgical Treatment of ESCC}

The approach of surgical therapy in patients with squamous cell carcinoma of the esophagus is influenced by the location of the tumor, the extent of metastatic spread, and the functional status of the patient. The treatment modality for a patient with ESCC located in the cervical region is administration of neoadjuvant radiochemotherapy followed by consecutive partial esophagectomy with interposition of a free jejunum transplant with a microvascular anastomosis [32]. This is in contrast to patients with ESCC located in the thoracic region which are surgically approached by performing a transthoracic Ivor Lewis esophagectomy or a transhiatal approach with an enlarged esophageal resection [33]. A study comparing these two surgical approaches, the transhiatal approach and the transthoracic esophageal resection, revealed similar survival rates with a positive trend towards a transthoracic resection [34]. A subgroup analysis regarding the effectiveness of both surgical procedures reported a difference regarding the lymph node involvement. The patients with $\mathrm{pN} 0$ lymph node status proved to have similar survival rates using both surgical procedures, whereas patients with $\mathrm{pN} 1$ lymph node status did not demonstrate a benefit from transthoracal resections. During surgery, dissection of the V. azygos is not necessary due to only $8 \%$ of patients having metatastic spread in this area [35].

\section{VIEW FOR THE FUture}

Even with optimal radiation and chemotherapy treatment and consecutive surgical resection, only 40-60\% of all patients with advanced tumor stage are reported to be cured. Furthermore this percentage varies with tumor stage.

Due to the limited improvement of chemotherapeutic agents, the responsibility for increased tumor control relies heavily on perfecting radiation techniques and surgical procedures. To optimize treatment in the 
future, additional treatment with advanced application of small molecules and administration of antibodies (targeted therapies) should be tested. These new treatment modalities are promising as shown by the effective inhibition of the epidermal growth factor receptor (EGFR), which is found to be over-expressed in more than $90 \%$ of patients with ESCC. Mutations of the K-RAS-genes, which revealed to predict a therapy-failure in colorectal cancer, are only rarely observed in squamous cell carcinoma of the esophagus [36]. EGFR antibodies have been reported to work synergistically in patients with ESCC and are commonly used to enhance the effect of radiochemotherapy [36]. Generally, an early response determination should be achieved as this would be beneficial for avoidance of treatment induced morbidity in these patients. The aim should be to achieve a molecular biological pre-therapeutic prediction. Future clinical research should be focused on determination of response prediction by biopsy testing. This standard of care is most notably used in breast cancer patients according to the results of translational research adopted for use in the clinical decision [37]. There are multiple studies evaluating molecular biological for markers such as p53, EGFR, ATM und CHK2 or Cyclin D1 in ESCC patients, however, they have limited relevance in everyday clinical care [38, 39]. Perioperative mortality represents a significant factor for the long-term survival of patients with esophageal carcinoma. Increased efforts and attention should be focussed to improve surgical treatment of these patients with the goal to decrease perioperative mortality. Proper patient selection by the physician under assistance of an interdisciplinary tumor board is also advised. Furthermore, surgical procedures of the esophagus should be limited to only highly qualified centers that are certified in this speciality.

\section{REFERENCES}

1. Jemal A, Murray T, Ward E et al (2005) Cancer statistics. CA Cancer J Clin 55(1):10-30

2. Matuschek C, Bölke E, Peiper M,. Knoefel WT, Budach W, Erhardt A, Scherer A, Gerber PA, Buhren BA, Gattermann N, Baldus SE, Rusnak E, Shukla V, Orth K. The role of neoadjuvant and adjuvant treatment for adenocarcinoma of the upper gastrointestinal tract. Eur J Med Res. 2011 June 21; 16(6): 265-274

3. Siewert JR, Feith M, Stein H (2005) Esophagectomy as therapeutic principle for squamous cell esophageal cancer. Chirurg 76:1033-1043

4. Siewert JR, Feith M, Werner M, Stein H (2000) Adenocarcinoma of the esophagogastric junction. Results of surgical therapy based on anatomical/topographic classification in 1,002 consecutive patients. Ann Surg 232:353-361

5. Kelsen DP, Ginsberg R, Pajak TF et al (1998) Chemotherapy followed by surgery compared with surgery alone for localized esophageal cancer. N Engl J Med 339: 1979 1984

6. Brücher BLDM, Swisher S, Königsrainer A et al (2009) Response to preoperative therapy in upper gastrointestinal cancers. Ann Surg Oncol 16(4):878-886

7. Brücher BLDM (2009) Plattenepithelkarzinom des Ösphagus. Chirurg 80: 1011-1018

8. Urschel JD, Vasan H (2003) A meta-analysis of randomized controlled trials that compared neoadjuvant chemoradiation and surgery with surgery alone for resectable esophageal cancer. Am J Surg 185:538-543
9. Fiorica F, Di Bona D, Schepis A et al (2004) Preoperative chemoradiotherapy for oesophageal cancer: a systematic review and meta-analysis. Gut 53:925-930

10. Malthaner RA, Wong R, Rumble RB (2004) Neoadjuvant or adjuvant therapy for respectable esophageal cancer: a systematic review and meta-analysis. BMC Med 2:3551

11. Stuschke M, Sarbia M (2004) Neoadjuvante Radiochemotherapie und Responseprädiktion. Onkologe 10:11791190

12. Greer SE, Goodney PP, Sutton JE et al (2005) Neoadjuvant chemoradiotherapy for esophageal carcinoma: a meta-analysis. Surgery 137:172-177

13. Gebski V, Burmeister B, Smithers BM et al (2007) Survival benefits from neoadjuvant chemoradiotherapy or chemotherapy in oesophageal carcinoma: a meta-analysis. Lancet Oncol 8:226-234

14. Schwartz GK, Winter K, Minsky BD et al (2009) Randomized phase II trial evaluating two paclitaxel and cisplatin-containing chemoradiation regimens as adjuvant therapy in resected gastric cancer (RTOG-0114). J Clin Oncol 27:1956-1962

15. Stahl M, Stuschke M, Lehmann N et al (2005) Chemoradiation with and without surgery in patients with locally advanced squamous cell carcinoma of the esophagus. J Clin Oncol 23:2311-2317

16. Stahl M, Wilke H, Lehmann N et al (2008) Long-term results of a phase III study investigating chemoradiotherapy with and without surgery in locally advanced squamous cell carcinoma of the esophagus. J Clin Oncol 26 [Suppl]:220 (Abstr 4530)

17. Bedenne L, Michel P, Bouche O et al (2007) Chemoradiation followed by surgery compared with chemoradiation alone in squamous cancer of the esophagus: FFCD 9102. J Clin Oncol 25:1160-1168

18. Therasse P, Arbuck SG, Eisenhauer EA et al (2000) New guidelines to evaluate the response to treatment in solid tumors. European Organization for Research and Treatment of Cancer, National Cancer Institute of the United States, National Cancer Institute of Canada. J Natl Cancer Inst 92(3):205-216

19. Beer AJ, Wieder HA, Lordick F et al (2006) Adenocarcinomas of esophagogastric junction: multi-detector row CT to evaluate early response to neoadjuvant chemotherapy. Radiology 239(2):472-480

20. Brücher BLDM, Stein HJ, Zimmermann F et al (2004) Responders benefit from neoadjuvant radiochemotherapy in esophageal squamous cell carcinoma: results of a prospective phase-II trial. Eur J Surg Oncol 30(9):963-971

21. Port JL, Lee PC, Korst RJ et al (2007) Positron emission tomographic scanning predicts survival after induction chemotherapy for esophageal carcinoma. Ann Thorac Surg 84:393-400

22. Hayano K, Okazumi S, Shuto K et al (2007) Perfusion CT can predict the response to chemoradiation therapy and survival in esophageal squamous cell carcinoma: initial clinical results. Oncol Rep 18(4):901-908

23. Wittekind C, Compton CC, Greene FL, Sobin LH (2002) TNM residual tumor classification revisited. Cancer 94:2511-2516

24. Mandard AM, Dalibard F, Mandard JC et al (1994) Pathologic assessment of tumor regression after preoperative chemoradiotherapy of esophageal carcinoma. Clinicopathologic correlations. Cancer 73(11):2680-2686

25. Chang F, Deere H, Mahadeva U, George S (2008) Histopathologic examination and reporting of esophageal carcinomas following preoperative neoadjuvant therapy: practical guidelines and current issues. Am J Clin Pathol 129(2):252-262

26. Schneider PM, Baldus SE, Metzger R et al (2005) Histomorphologic tumor regression and lymph node metas- 
tases determine prognosis following neoadjuvant radiochemotherapy for esophageal cancer: implications for response classification. Ann Surg 242(5):684-692

27. Becker K, Mueller JD, Schulmacher C, Ott K et al (2003) Histomorphology and grading of regression in gastric carcinoma treated with neoadjuvant chemotherapy. Cancer 98:1521-1530

28. Brücher BLDM, Becker K, Lordick F et al (2006) The clinical impact of histopathologic response assessment by residual tumor cell quantification in esophageal squamous cell carcinomas. Cancer 106(10):2119-2127

29. Swisher S, Erasmus J, Masih M et al (2004) 2-Fluoro-2deoxy-D-glucose positron emission tomography imaging is predictive of pathologic response and survival after preoperative chemoradiation in patients with esophageal carcinoma. Cancer 101(8):1776-1785

30. Wieder H, Brücher BLDM, Zimmermann F et al (2004) Time course of tumor metabolic activity during chemoradiotherapy of esophageal squamous cell carcinoma and response to treatment. J Clin Oncol 22(5):900-908

31. Taylor MD, Smith PW, Brix WK et al (2009) Correlations between selected tumor markers and fluorodeoxyglucose maximal standardized uptake values in esophageal cancer. Eur J Cardiothorac Surg 35(4):699-705

32. Ott K, Lordick F, Molls M et al (2009) Limited resection and free jejunal graft interposition for squamous cell carcinoma of the cervical oesophagus. Br J Surg 96(3):258266

33. Lewis I (1945) Carcinoma of the esophagus. Radical resection with oesophagogastrostomy for a midthoracic growth by right transpleural approach. J R Soc Med 38: 482-483

34. Hulscher JB, van Sandick JW, de Boer AG et al (2002) Extrended thransthoracic resection compared with limited transhiatal resection for adenocarcinoma of the esophagus. N Engl J Med 347(21):1662-1669

35. Omloo JM, Lagarde SM, Hulscher JB et al (2007) Extended transthoracic resection compared with limited transhiatal resection for adenocarcinoma of the $\mathrm{mid} /$ distal esophagus: five-year survival of a randomized clinical trial. Ann Surg 246(6):992-1000

36. Bonner JA, Harari PM, Giralt J et al (2006) Radiotherapy plus Cetuximab for squamous-cell carcinoma of the head and neck. N Engl J Med 354:567-578
37. Perez EA, Bawejy M (2008) HER2-positive breast cancer: current treatment strategies. Cancer Invest 26(6):545-552

38. Sarbia M, Pühringer-Oppermann F, Brücher BLDM (2007) The predicitve value of molecular markers ( $\mathrm{p} 53$, EGFR, ATM, CHK2) in multimodal treated esophageal squamous cell carcinoma. Br J Cancer 97(19):1404-1408

39. Brücher BLDM, Keller G, Werner M et al (2009) Using Q-RT-PCR to measure Cyclin D1, TS, TP, DPD, and Her-2/neu as predictors for response, survival and recurrence in patients with esophageal squamous cell carcinoma following radiochemotherapy. Int J Colorectal Dis 24(1):69-77

40. Schuler PJ, Börger V, Bölke E, Habermehl D, Matuschek C, Wild CA, Greve J, Bas M, Schilling B, Bergmann C, Trellakis S, Budach W, Gauler T, Brandau S, Lang S, Whiteside TL, Sorg RV, Hoffmann TK. Dendritic cell generation and CD4+ CD25high FOXP3+ regulatory $\mathrm{t}$ cells in human head and neck carcinoma during radiochemotherapy. Eur J Med Res. 2011 Feb 24;16(2):57-62.

41. Schauer M, Peiper M, Theisen J, Knoefel W. Prognostic factors in patients with diffuse type gastric cancer (linitis plastica) after operative treatment. Eur J Med Res. 2011 Jan 27;16(1):29-33.

42. Schuler PJ, Trellakis S, Greve J, Bas M, Bergmann C, Bölke E, Lehnerdt G, Mattheis S, Albers AE, Brandau S, Lang S, Whiteside TL, Bier H, Hoffmann TK. In vitro chemosensitivity of head and neck cancer cell lines. Eur J Med Res. 2010 Aug 20;15(8):337-44.

Received: May 3,2011 / Accepted: June 1, 2011

Address for correspondence:

PD Dr. med. Edwin Bölke

Klinik für Strahlentherapie und Radiologische Onkologie

Heinrich-Heine-Universität Düsseldorf

Moorenstraße 5

40225 Düsseldorf

Germany

E-mail: Boelke@med.uni-duesseldorf.de 